\title{
ACÚMULO DE POLICLORETOS DE BIFENILA NA POPULAÇÃO DO GRANDE SÃO PAULO, BRASIL*
}

\author{
Diogo Pupo Nogueira** \\ José Maria Pacheco de Souza*** \\ Sérgio Colacioppo** \\ Jorge da Rocha Gomes** \\ Joselito Bomfim Brandão** \\ Marlene Lopes Assis de Souza**
}

\begin{abstract}
NOGUEIRA, D. P. et al. Acúmulo de policloretos de bifenila na população do Grande São Paulo, Brasil. Rev. Saúde públ., S. Paulo, 21:279-90, 1987.

RESUMO: Os policloretos de bifenila (PCBs), cuja utilização iniciou-se em 1930 em sistemas fechados (capacitores e transformadores elétricos) foram observados no ambiente em 1966, atingindo a cadeia alimentar e acumulando-se no tecido subcutâneo de seres humanos; ao mesmo tempo, foi possível mostrar que esses produtos químicos são potencialmente nocivos para a saúde humana. Como em 1976 os PCBs foram ercontrados no tecido gorduroso de pequena amostra da população brasileira, decidiu-se fazer um estudo mais amplo, analisando-se por cromatografia de fase gasosa a gordura do tecido celular subcutâneo de 189 pessoas, de ambos os sexos, que tinham sofrido morte violenta. Foram encontrados níveis médios de 0,8661 ppm em indivíduos do sexo masculino, que apresentaram aumento linear das concentraçóes de acordo com a idade, o que não foi observado entre os do sexo feminino, onde os valores médios de acúmulo foram de 1,4530 ppm. Do total de amostras, $12,7 \%$ apresentaram níveis superiores a $1,95 \mathrm{ppm}$. E discutida a importância do achado e são assinaladas as medidas de proteção da população brasileira exposta aos PCBs.
\end{abstract}

UNITERMOS: Compostos de policlorobifenil, análise. Poluição ambiental. Contaminação química de alimentos.

\section{INTRODUÇÃO}

Os policloretos de bifenila (PCBs) são produtos químicos provenientes da substituição de um ou mais átomos de hidrogênio por átomos de cloro na estrutura do radical bifenil. Sob a forma sólida ou líquida ${ }^{33}$, são química e termicamente estáveis e excelentes dielétricos, sendo por isso utilizados em transformadores e capacitores elétricos e como trocadores de calor $^{19,33,47}$. São ainda usados em fluídos hidráulicos, na composição de tintas e lacas ${ }^{5}$, em papéis copiativos sem carbono ${ }^{61}$, como lubrificantes $^{44}$, em tintas de impressão ${ }^{44}$, bombas a vácuo e capeamento ${ }^{47}$. São extremamente estáveis e, segundo Smith ${ }^{59}$, se degradam apenas $50 \%$ após vinte anos.

Os PCBs foram criados para serem utilizados em sistemas fechados. No entanto, em
1969 Jensen $^{26}$ encontrou PCBs em resíduos de peixes, aves e seres humanos, na Suécia e em outros paises europeus. Estudos posteriores evidenciaram que os PCBs tinham se transformado em poluidores globais e que seus níveis tinham se aproximado, senão ultrapassado, aqueles do DDT e demais inseticidas organoclorados. Tais achados foram especialmente preocupantes porque, ao contrário de inseticidas organoclorados, os PCBs nunca tinham sido introduzidos intencionalmente no meio ambiente e, não obstante, tornaram-se um problema de saúde pública?.

A partir da descoberta de Jensen ${ }^{26}$, $O$ interesse pelos PCBs cresceu e uma série de estudos - experimentais em animais de laboratório, clínicos e epidemiológicos - vieram

* Pesquisa financiada pela Fundação de Amparo à Pesquisa do Estado de São Paulo (FAPESP) Processo n. ${ }^{\circ} 77 / 1120$-I e com apoio técnico da Fundação Jorge Duprat Figueiredo de Segurança e Medicina do Trabalho.

* Departamento de Saúde Ambiental da Faculdade de Saúde Pública da Universidade de São Paulo Av. Dr. Arnaldo, 715 - 01255 - Sáo Paulo, SP - Brasil.

*** Departamento de Epidemiologia da Faculdade de Saúde Pública da Universidade de São Paulo Av. Dr. Arnaldo, 715 - 01255 - São Paulo, SP - Brasil. 
mostrar que esses produtos químicos ofereciam sérios riscos à saúde humana.

Estudos realizados em ratos alimentados com dietas contendo $\mathrm{PCBs}$ vieram mostrar que o fígado era um órgão alvo. Assim, foram observadas alterações estruturais e degenerativas das células hepáticas ${ }^{5}$; degeneração celular e atrofia centrobolular ${ }^{38}$; hipertrofia de células hepáticas, com inclusões no citoplasma, pigmento marrom nas células de Kupfer, acúmulo de líquidos nas células e adenofibrose ${ }^{28}$; aumento de colesterol hepático ${ }^{71}$; metaplasia e adenocarcinoma hepático ${ }^{41}$; quadruplicação do efeito cancerígeno da dietilnitrosamina ${ }^{49}$; necrose focal ${ }^{27}$; carcinoma hepatocelular ${ }^{58}$; inibição da uroporfirinogenocarboxilase do fíga$\mathrm{go}^{43}$; aumento da metaplasia gastrointestinal seguida de adenocarcinoma e de adenoma hepatocelular ${ }^{62}$; aumento do peso e do colesterol ${ }^{12}$ e aumento da 3-hidroxi-3-metilglutamil coezima A redutase ${ }^{25}$.

Também em outros animais observaram-se alterações hepato-tóxicas quando em suas dietas figuravam os PCBs. Assim, em coelhos, foi observada hipertrofia dos hepatócitos centrolobulares ${ }^{45}$; em furöes, aumento do citocromo microssomal ${ }^{3}$ e em primatas (Macaca mulatta), hipertrofia celular e proliferação do retículo endoplasmático.

Verificou-se ainda que os PCBs afetam a pele dos animais de experiência. Assim, foram encontradas lesões semelhantes ao do clorác$n \mathrm{e}^{38,43} \mathrm{e}$ alopécia ${ }^{43} \mathrm{em}$ ratos e coelhos, assim como edema subcutâneo em aves ${ }^{52}$.

Animais de experiência também apresentaram outras patologias quando alimentados com dietas contendo PCBs. Assim, camundongos fêmeas prenhes e recebendo os produtos no $6 .^{\circ}$ e $15 .^{\circ}$ dias de gestação davam nascimento a filhos com graves efeitos teratogênicos ${ }^{36}$. Por outro lado, verificou-se que ratos que recebiam tais dietas apresentavam diminuição da resistência a infecções sistêmicas e locais causadas por vírus e bactérias ${ }^{23}$.

Seres humanos expostos aos PCBs, quer através de alimentos ou por razāo ocupacional, apresentavam uma série de problemas de saúde. Merecem destaque o aumento da indução de enzimas microssômicas do fígado ${ }^{21}$; maior incidência de toxemia gravídica ${ }^{\text {t4 }}$; de prematuridade $^{65}$; presença de lesões acneiformes ${ }^{56}$; lesões difusas da pele ${ }^{46}$; aumento da incidência de melanoma e de câncer do pâncreas ${ }^{51}$; maior incidência de cirrose hepática e de câncer do fígado ${ }^{51}$; alteração das provas de função hepática ${ }^{10}$; alterações congênitas de crianças cujas mães tinham ingerido alimento com teores de resíduos de $\mathrm{PCBs}$, constantes de conjuntivite, prurido cutâneo, maior incidência de cárie dentária, fragilidade ungueal, pigmentação marrom da pele, esmalte dentário manchado ${ }^{72}$, prematuridade e menor peso ao nascer ${ }^{60}$, grau moderado de retardo mental ${ }^{24}$ e depressāo do sistema imunol6gico ${ }^{53}$.

Em 1968 cerca de 6.611 pessoas ingeriram, no Japão, óleo de arroz contaminado por PCBs e o mesmo ocorreu em 1979 em Formosa, quando 2.000 pessoas foram afetadas ${ }^{14,31}$. As pessoas que se alimentaram com esse óleo contaminado apresentaram acentuação folicular, erupção acneiforme, pigmentação da pele e das unhas e hipersecreção das glândulas de Meibomius. As crianças nascidas após esse acidente alimentar apresentavam pele, unhas e gengivas de cor marrom, epífora acentuada ${ }^{31}$, fontanelas e suturas amplamente abertas ${ }^{39}$ e depressão imunológica ${ }^{53}$.

Trabalhadores expostos a concentrações elevadas de PCBs após fogo e/ou explosão de transformadores e de capacitores apresentaram moderada elevação das enzimas hepáticas, mitocondria pleomorfa, mitocondria gigante, aumento da densidade dos grânulos mitocôndricos, gotículas de gordura nas células hepáticas, retículo endoplasmático ligeiramente dilatado $0^{55}$, neuropatia periférica sensorial com diminuição da velocidade de condução nervosa ${ }^{14}$, parestesia cutânea ${ }^{57}$, câncer cutâneo (um caso) ${ }^{54} \mathrm{e}$ alterações da função respiratória do tipo obstrutivo $0^{32}$.

A introdução dos PCBs no organismo humano faz-se através do $a^{35}$, da água ${ }^{18,26,34,66}$, do solo $^{22}$ e, especialmente, dos alimentos tais como carne, leite, ovos, hortaliças, peixes,

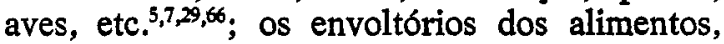
fabricados com papel contendo PCBs que é reciclado, são fonte importante de contaminação alimentar ${ }^{1}$, assim como silos contaminados.

Um alimento cuja contaminação pelos PCBs tem importância é o leite humano. Devido ao fato das mães acumularem PCBs no seu organismo, há contaminação do leite $4,11,48,61,67,72$.

Os PCBs introduzidos no organismo humano depositam-se em tecidos que contenham elevado teor de gordura. Por essa razāo, o estudo do acúmulo dos PCBs no organismo das populações geralmente é feito pela análise do tecido celular subcutâneo ${ }^{70}$ obtido através de atos cirúrgicos e/ou necrópsias ${ }^{37,61,73}$, mas também pela análise do sangue $e^{20}$ ou plasma ${ }^{48}$, sendo geralmente preferido o estudo do tecido 
celular subcutâneo. O método de análise preferido é o da cromatografia de fase gasosa.

A Organização Mundial de Saúde ${ }^{70}$ observou em vários países valores médios de $1 \mathrm{mg} / \mathrm{kg}$, e outros estudos mostraram valores de 0,103 ppm (partes por milhão) na Alemanha ${ }^{61}$; valores superiores a $2 \mathrm{ppm}$ foram observados em $5,2 \%^{73}, 5,6 \%^{50}$ e $31 \%$ de norte-americanos. Estudos feitos dosando-se os PCBs do plasma revelaram 3,99 $\mathrm{ppb}$ (partes por bilhão) em Israel ${ }^{48}$ e 4 a $8 \mathrm{ng} / \mathrm{ml}$ na população geral dos Estados Unidos ${ }^{29}$.

No Brasil, em 1976, Wassermann e col. ${ }^{63}$ fizeram uma pesquisa sobre a deposição de inseticidas organoclorados em neoplasias da mama e em tecido adjacente aparentemente normal em pacientes do sexo feminino de hospital de São Paulo; ao se proceder à análise cromatográfica das amostras de neoplasia da mama (5 casos) os níveis médios de PCBs encontrados foram de $0,972 \mathrm{ppm}$, com extremos de 0,28 a 1,44 ppm; no grupo controle (9 casos) os valores médios foram de 0,7658 ppm com extremos de 0,38 a 1,02 ppm.

Esse primeiro estudo ${ }^{63}$ veio mostrar que, a exemplo do que havia sido verificado em outros países do mundo, os PCBs também tinham atingido o meio ambiente e já se encontravam depositados no organismo de brasileiros. $O$ número relativamente pequeno de amostras obtidas na ocasião, decorrente do fato de que o estudo estava sendo feito somente em pacientes portadores de neoplasias mamárias, não permitiu que os resultados fossem inferidos para a população da capital do Estado de São Paulo, onde se localizava o hospital, e muito menos para a população brasileira. Por essa razão, ficou evidenciada a necessidade de um estudo maior, que pudesse avaliar de forma mais positiva quais os níveis de PCBs na população da cidade de São Paulo.

O presente trabalho tem, portanto, o objetivo de estudar o acúmulo dos PCBs no tecido celular subcutâneo de habitantes do Grande São Paulo.

\section{MATERLAL E METODOS}

\section{Amostragem}

Foram obtidas 189 amostras de tecido adiposo por ocasião de autópsias de vítimas de morte violenta, realizadas no Instituto Médico Legal da Secretaria de Segurança Pública de São Paulo, seqüencialmente. Tais amostras foram coletadas em frascos de vidro, previamente lavados e enxugados com n-Hexano livre de residuos, fechados com tampa de teflon e contendo solução de formol a $1,0 \%$.

\section{Métodos Analíticos}

O método analítico utilizado foi a combinação das propostas de Wood ${ }^{69}$, Erney ${ }^{17}$, Berg e col. ${ }^{6}$ e De Kok e col..$^{15}$. Em resumo, o método utilizado está a seguir descrito.

Cerca de um grama de tecido adiposo foi fragmentado e dissolvido em n-Hexano a quente, misturado com celite e, a seguir, empacotado em uma pequena coluna de vidro. Os resíduos de pesticidas organoclorados e de policloretos de bifenila foram eluídos com dimetil sulf6xido. O eluato foi adsorvido em coluna de florisil e os resíduos foram recuperados do dimetil sulfóxido por eluição com n-Hexano.

O eluato foi concentrado e os policloretos de bifenila presentes foram separados dos pesticidas organoclorados por passagem em coluna de sílica-gel.

O eluato obtido da coluna de sílica-gel foi concentrado e o resíduo decaclorado com pentacloreto de antimônio a quente. $O$ derivado decaclorado foi extraído com n-Hexano e concentrado a volume apropriado para determinação cromatográfica.

As amostras foram cromatografadas em um cromatógrafo a gás CG-370, com detector de captura de eletrons (de níquel). A identificação foi efetuada em coluna QF-1 Anakron ABS $3 \%$ e a quantificação em coluna OV-210 $5 \%$ em Cromosorb $\mathrm{Q}$. O padrão para cálculo foi o Aroclor 1254, fornecido pela "Environmental Protection Agency" dos Estados Unidos.

\section{Análise Estatística dos Resultados}

A análise foi exploratória, partindo-se da hipótese de trabalho que admitiu um acúmulo de contaminação à medida que a idade avança e uma contaminação zero ao nascer. $O$ modelo proposto foi o mais simples possível, ou seja, um crescimento linear a partir da contaminação zero ao nascer. Cada sexo foi analisado em separado; trabalhou-se com grupos etários de 10 em 10 anos, usando-se a média das idades em cada grupo como referência para o conjunto do respectivo intervalo; como a informação obtida foi em anos completos, à idade média calculada acrescentou-se 0,5 ano para o devido ajuste para a variável contínua. A idade mínima foi zero e a máxima considerada foi 59 anos completos, não se tendo considerado na análise os valores obtidos de cadáveres de pessoas com 60 anos ou mais, que totalizam 14 do sexo feminino e 10 do sexo masculino. A investigação teve a seguinte sequiência: identificação e eliminação de possíveis valores estatisticamente aberrantes de contaminação, independentemente da idade: cálculo do valor 
médio de PCBs em cada grupo etário: alocação das médias e dos acúmulos individuais em gráficos, para inspeção visual da possível tendência linear do fenômeno; ajuste de reta de regressão, se razoável com o modelo; estabelecimento de limites de predição, independentemente da razoabilidade, para escolher indivíduos com contaminação além desses limites a serem considerados como casos em possível estudo de investigação analítica tipo caso-controle. Os textos estatísticos de referência foram Berquó e col. ${ }^{8}$ e Montgomery e Peck ${ }^{40}$.

\section{RESULTADOS}

Foi obtido um total de 189 amostras de tecido celular subcutâneo e as Tabelas 1 e 2

TABELA 1

Número e percentagem de indivíduos segundo idade e sexo

\begin{tabular}{|c|c|c|c|c|c|c|}
\hline \multirow{3}{*}{$\begin{array}{l}\text { Grupo etário } \\
\text { (anos) }\end{array}$} & \multicolumn{4}{|c|}{ Sexo } & \multirow{2}{*}{\multicolumn{2}{|c|}{ Tota 1}} \\
\hline & \multicolumn{2}{|c|}{ Masculino } & \multicolumn{2}{|c|}{ Feminino } & & \\
\hline & N. ${ }^{\circ}$ & $\%$ & N. ${ }^{\circ}$ & $\%$ & N. ${ }^{\circ}$ & $\%$ \\
\hline $\begin{array}{r}0-9 \\
10-19 \\
20-29 \\
30-39 \\
40-49 \\
50-59\end{array}$ & $\begin{array}{l}17 \\
23 \\
25 \\
15 \\
17 \\
11\end{array}$ & $\begin{array}{l}15,74 \\
21,30 \\
23,15 \\
13,89 \\
15,74 \\
10,18\end{array}$ & $\begin{array}{r}14 \\
21 \\
20 \\
5 \\
12 \\
9\end{array}$ & $\begin{array}{r}17,28 \\
25,93 \\
24,69 \\
6,17 \\
14,82 \\
11,11\end{array}$ & $\begin{array}{l}31 \\
44 \\
45 \\
20 \\
29 \\
20\end{array}$ & $\begin{array}{l}16,41 \\
23,28 \\
23,81 \\
10,58 \\
15,34 \\
10,58\end{array}$ \\
\hline Total & 108 & 100,00 & 81 & 100,00 & 189 & 100,00 \\
\hline $\begin{array}{l}\text { Média } \\
\text { (anos de idade) } \\
\text { Desvio padrão } \\
\text { (anos de idade) }\end{array}$ & & & & & & \\
\hline
\end{tabular}

TABELA 2

Número de indivíduos segundo nível de PCBs e sexo

\begin{tabular}{|c|c|c|c|c|}
\hline \multirow{2}{*}{ Nível } & \multirow{2}{*}{\multicolumn{2}{|c|}{ de PCB (ppm) }} & \multicolumn{2}{|c|}{ Sexo } \\
\hline & & & Masculino & Feminino \\
\hline 0 & - & 0,15 & 14 & 15 \\
\hline 0,15 & - & 0,30 & 23 & 5 \\
\hline 0,30 & - & 0,45 & 14 & 10 \\
\hline 0,45 & L & 0,60 & 15 & 10 \\
\hline 0,60 & - & 0,75 & 8 & 5 \\
\hline 0,75 & 1 & 0,90 & 4 & 5 \\
\hline 0,90 & - & 1,05 & 4 & 4 \\
\hline 1,05 & - & 1,20 & 2 & 3 \\
\hline 1,20 & $\longrightarrow$ & 1,35 & 5 & 2 \\
\hline 1,35 & - & 1,50 & 2 & 1 \\
\hline 1,50 & $\ldots$ & 1,65 & 2 & 3 \\
\hline 1,65 & 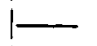 & 1,80 & 2 & 3 \\
\hline 1,80 & $\ldots$ & 1,95 & 1 & 3 \\
\hline 1,95 & 工 & 2,20 & 3 & 1 \\
\hline 2,20 & - & 2,35 & 2 & 1 \\
\hline 2,68 & 3,08 & 3,16 & & \\
\hline 3,60 & $\begin{array}{r}3,84 \\
10,79\end{array}$ & 4,13 & 7 & \\
\hline $\begin{array}{l}2,85 \\
3,45 \\
6,94\end{array}$ & $\begin{array}{r}3,07 \\
4,71 \\
7,13 \\
23,13\end{array}$ & $\begin{array}{l}3,40 \\
5,70 \\
9,65\end{array}$ & 10 & \\
\hline Total & & & 108 & 81 \\
\hline $\begin{array}{l}\text { médi } \\
\text { desvi }\end{array}$ & -padrã & & $\begin{array}{l}0,8661 \\
1,2824\end{array}$ & $\begin{array}{l}1,4530 \\
2,9746\end{array}$ \\
\hline
\end{tabular}

apresentam as distribuiçōes de idades e de acúmulo segundo sexo, com as médias e desvios-padrão.

No sexo masculino, após inspeção, admitiu-se como possível valores aberrantes 10,79 ppm e 4,13 ppm de PCBs. Eliminando-se tais observações, recalculando-se a média e o desvio-padrão e fazendo-se então o devido teste, confirma-se estatisticamente a hipótese. No sexo feminino o mesmo procedimento definiu 23,12 ppm e 9,65 de PCBs como aberrantes. A Tabela 3 descreve tais achados e apresenta resumo dos resultados estatísticos subsequientes.

A Figura 1, referente ao sexo masculino, sugere que uma reta de regressão, passando pela origem, pode se ajustar razoavelmente às médias de concentração de PCBs segundo os grupos etários. A equação obtida é $\mathrm{y}=0,0259$ $\mathrm{x}$, onde y é a média estimada de concentração e $x$ é a idade.

Já a Figura 2, referente ao sexo feminino, indica que o fenômeno de acúmulo, neste caso, não pode ser explicado via crescimento linear segundo idade; a construção de uma reta de regressão $\mathrm{y}=0,0392 \mathrm{x}$ teve por finalidade marcar esta dificuldade e auxiliar na identificação de possíveis "casos" via limite de predição. Análises de variância da regressão pela origem confirmam os resultados. 
TABELA 3

Resumo das estatísticas segundo sexo

\begin{tabular}{|c|c|c|}
\hline \multirow{2}{*}{ Estatísticas } & \multicolumn{2}{|c|}{ Sexo } \\
\hline & Masculino & Feminino \\
\hline \multirow{2}{*}{$\begin{array}{l}\text { Número inicial de observaçóes } \\
\text { Valores considerados aberrantes e eliminados } \\
\text { das análises (em ppm) } \\
\text { Número de observações consideradas nas análises } \\
\text { Média eliminando os valores } \quad\left\{\begin{array}{l}\text { idade } \\
\text { aberrantes }\end{array}\right.\end{array}$} & 108 & 81 \\
\hline & $\begin{array}{cc}10,79 & 4,13 \\
106 & \\
27,03 & \\
0,7417 & \end{array}$ & $\begin{array}{cc}23,13 & 9,65 \\
79 & \\
25,63 & \\
1,0748 & \end{array}$ \\
\hline$\left\{\begin{array}{l}\text { idade } \\
\text { PCB }(\mathrm{ppm})\end{array}\right.$ & $\begin{array}{l}15,25 \\
0,7880\end{array}$ & $\begin{array}{l}16,72 \\
1,4299\end{array}$ \\
\hline \multirow[t]{2}{*}{ Regressão completa } & $\begin{array}{l}0,173826 \\
0,021010\end{array}$ & $\begin{array}{l}0,237337 \\
0,032678\end{array}$ \\
\hline & $\begin{array}{c}1,2110 \\
\text { (não significante) }\end{array}$ & $\begin{array}{c}0,8626 \\
\text { (não significante) }\end{array}$ \\
\hline $\begin{array}{l}\text { Regressão pela origem } \\
\text { Desvio-padrão de } b \\
\text { Teste } \tau \text { de } \beta \\
\text { Valores acima dos limites de predição } \\
\text { (entre parêntesis o grupo etário) }\end{array}$ & 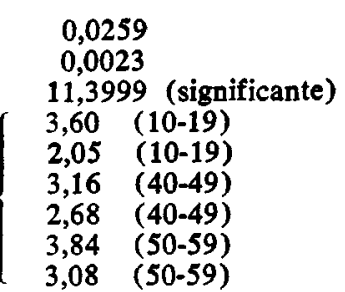 & $\begin{array}{l}3,07(0-9) \\
7,13(40-49) \\
9,94(40-49) \\
5,70(40-49) \\
4,71(40-49)\end{array}$ \\
\hline
\end{tabular}

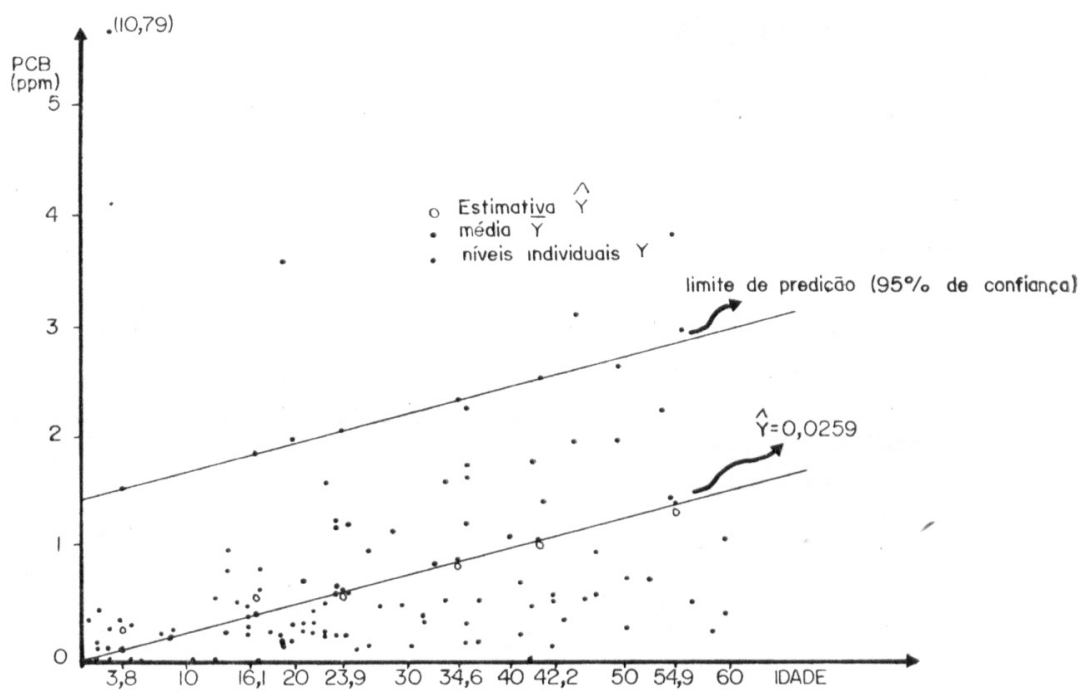

Fig. 1 - Níveis individuais, médios e estimados por regressão pela origem de PCB, segundo médias de idade. Sexo masculino.

Os limites (superiores) de predição, com coeficientes de $95 \%$, permitem selecionar 6 possíveis "casos" no sexo masculino, além dos 2 considerados inicialmente como valores aberrantes. No sexo feminino 5 "casos" são detectados por esse processo. Seus níveis de acúmulo aparecem nas Figuras 1 e 2.

\section{COMENTÁRIOS}

Decidiu-se obter amostras de tecido celular subcutâneo de habitantes do Grande São Paulo que tivessem sofrido morte violenta; a escolha desse tipo de morte teve por objetivo evitar-se
- estudo de pessoas sabidamente portadoras de doenças agudas ou crônicas, como ocorrera no estudo de Wassermann e col. ${ }^{63}$, para evitar a possibilidade de que esses estados mórbidos pudessem ter influência sobre os níveis de acúmulo.

Teria sido ideal o exame de maior número de indivíduos, como originalmente tinha sido previsto; no entanto, mesmo prolongando-se $o$ tempo de estudo muito além do calculado, não foi possível obter-se mais amostras, além dos 189 indivíduos. As mesmas dificuldades rela- 


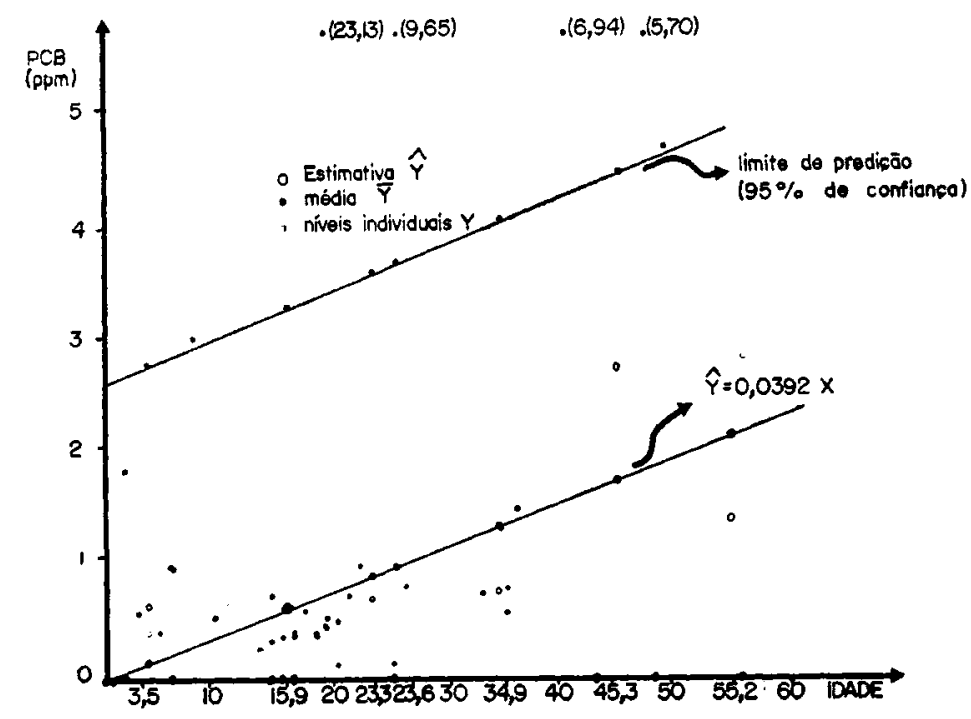

Fig. 2 - Níveis individuais, médios e estimados por regressão pela origem de PCB, segundo. médias de idade. Sexo feminino.

tadas por Moriaty ${ }^{42}$ e Anderson ${ }^{2}$ estiveram presentes neste trabalho.

É de se notar que mesmo em países altamente desenvolvidos, onde o nível de colaboração de todas as entidades envolvidas é muito maior, não tem sido possível a obtenção de grande número de amostras de tecido celular subcutâneo. Excetuando-se a pesquisa de Yobs ${ }^{73}$, que estudou 688 amostras de tecido celular subcutâneo durante atos cirúrgicos ou de autópsias decorrentes de acidentes em pessoas moradoras de 14 Estados norte-americanos, a maioria dos estudos foi feita com número de amostras inferior ao presente trabalho; assim, por exemplo, Mes e col. ${ }^{37}$, não obstante terem trabalhado com serviços médico-legais de nove províncias canadenses, não obtiveram mais do que 99 amostras de tecido celular subcutâneo proveniente de pessoas mortas por motivo de acidente; Wolf e col ${ }^{68}$ utilizaram 26 amostras e Chase e col. ${ }^{13}, 50$ amostras em seus respectivos estudos. Dessa forma, o número de indivíduos examinados no presente estudo é suficientemente elevado para permitir uma análise mais detalhada dos valores de acúmulo encontrados.

Note-se que a análise por cromatografia gasosa dos PCBs é extremamente delicada e exige rigor técnico muito maior do que, por exemplo, a análise de pesticidas organoclorados, o que constitui um obstáculo para o exame de número muito grande de amostras; isto explica porque na maioria dos estudos realizados a amostra analisada é relativamente pequena. No caso particular da pesquisa de $\mathrm{Yobs}^{73}$, teve este 0 auxílio de toda a máquina governamental norte-americana, tanto a nível federal como estadual.

O uso de cadáveres em investigação traz alguma limitação para a transposição dos resultados para indivíduos vivos. Não se consegue, no entanto, identificar possibilidade para vício de grande monta neste estudo de acúmulo dos PCBs. Estudos mais elaborados e mais difíceis de serem realizados em indivíduos vivos, provavelmente, confirmarão os presentes achados; nestes casos, porém, a obtenção de amostras de tecido celular subcutâneo, mesmo por intermédio de biópsia por agulha, oferece maiores dificuldades práticas, visto tratar-se de método invasivo que só pode ser utilizado com a permissão das pessoas examinadas, dentro dos princípios éticos que regem as pesquisas científicas, e essa permissão nem sempre é fácil de ser obtida.

A aplicação de modelo de regressão linear, em seus aspectos dependentes de pressuposiçōes quanto a distribuição de probabilidades, deve levar, ainda assim, a resultados com boa confiabilidade, bastante úteis ao raciocínio epidemiológico.

Um achado inesperado é a diferença de comportamento do acúmulo de $\mathrm{PCBs}$ entre indivíduos do sexo masculino e feminino, sendo maior neste, ao contrário do que tem sido observado em outras regiões do mundo. Assim, as mulheres apresentaram valor médio de $1,0748 \mathrm{ppm}$ de PCBs contra 0,7417 ppm em homens. No estudo que Wassermann e col.63 realizaram em mulheres brasileiras, não-portadoras de qualquer patologia evidente ( 5 pes- 
soas), os níveis de acúmulo encontrados foram de $0,7658 \mathrm{ppm}$, enquanto que nos casos de portadores de câncer da mama esse valor médio era de $0,9720 \mathrm{ppm}$, significantemente menor que o encontrado no presente caso.

É difícil dar-se uma explicação para o maior acúmulo de PCBs em indivíduos do sexo feminino. Uma hipótese seria a de que as mulheres ingeririam maior quantidade de alimentos com resíduos de PCBs que os homens, a exemplo do que foi observado por Wickizer e Brillant ${ }^{67}$. Como, no entanto, não existe no Brasil nenhum estudo sobre resíduos de PCBs em alimentos utilizados pela população brasileira, essa explicação permanece no terreno puramente hipotético.

No sexo masculino parece haver tendência a maior nível para mais elevada idade, quer observando-se valores individuais, quer observando-se as médias de acúmulo segundo a média de idade, o que não ocorre entre as mulheres. Tal fato foi observado também por Kreis e col. ${ }^{30}$ que afirmaram " ... os níveis médios de PCBs aumentaram com a idade..

$\mathrm{O}$ aumento dos níveis médios de $\mathrm{PCBs}$ com a idade ... sugere uma acumulação ao longo do tempo, maior exposição no passado ou uma repartição dos PCBs entre soro e tecido adiposo relacionado com a idade".

Já no sexo feminino, o nível de acúmulo parece oscilar, para mais ou para menos, em torno de algum valor intermediário, não se podendo argumentar com crescimento linear do acúmulo.

E lícito, pois, pensar-se em condições diferentes de exposição aos PCBs, propondo-se a seguinte hipótese do processo: nas idades iniciais homens e mulheres teriam o mesmo grau de exposição, decorrente possivelmente da alimentação com alimentos que contenham resíduos de PCBs, o que levaria a acúmulo semelhante uma vez que, quando crianças e adolescentes, os tipos de vida para ambos os sexos não são intrinsecamente diferentes quanto à exposição aos PCBs. Na vida adulta, porém, as atividades exercidas pelos homens levariam à exposição contínua (possivelmente de natureza ocupacional) aos $\mathrm{PCBs}$, elevando gradativamente o seu nível acumulado. Isto não ocorreria em geral com as mulheres, que apenas manteriam o nível de contaminação pronunciadamente maior que o do homem acumulado até a idade jovem.

Um modelo mais complexo a ser estudado, que depende, contudo, de dados bastante exatos e de difícil obtenção, é aquele que consi- deraria a época de entrada em uso dos PCBs no Brasil e, particularmente, no Grande São Paulo. Admitindo-se, para exemplificar, introdução em 1940, a contaminação acumulada se iniciaria aos zero anos de idade para aqueles nascidos nesse ano, mas aos 20 anos para os nascidos em 1920, ou seja, a regressão deveria considerar a idade inicial à exposição, algo semelhante aos modelos aplicados de incidência de câncer do pulmão e fumo.

Propõe-se também uma abordagem epidemiológica diferente da aqui empregada (corte transversal) para complementar a investigação entre exposição a PCBs e respectivo acúmulo. E o uso de estudo tipo caso-controle, aninhado na própria amostra utilizada no estudo tipo transversal. Dentro desta linha de trabalho é que se estabelecem as linhas de limites de predição, para ambos os sexos. Pode-se considerar como "caso" todo indivíduo cuja contaminação esteja acima dos limites, levando a um total de 6 indivíduos do sexo masculino e 5 do feminino. Para aumentar o número de casos, uma sugestão é utilizar acúmulos abaixo destes limites, mas os mais próximos possíveis, segundo idade. Como controle, seria interessante considerar "sem acúmulo" indivíduos com acúmulo menor do que a linha média de regressão. No sexo feminino alguma modificação seria necessária, devido a não linearidade do fenômeno.

Comparando-se os achados deste estudo, verifica-se que os niveis de $\mathrm{PCBs}$ encontrados na população do Grande São Paulo devem constituir motivo de preocupação. Realmente $12,70 \%$ dos casos mostram acúmulo superior a $1,95 \mathrm{ppm}$; tal valor é bastante maior que o observado em estudos semelhantes feitos em outros países. Assim, Yobs ${ }^{73}$ no seu estudo encontrou que apenas $5,2 \%$ das amostras apresentavam valores superiores a $2 \mathrm{ppm}$ e Price e Welch ${ }^{50}$ encontraram apenas $5,6 \%$ da população estudada com valores que excediam 2 ppm. Dessa forma, a população do Grande São Paulo apresenta um acúmulo ao nível de mais de $2 \mathrm{ppm}$ que é aproximadamente duas vezes maior do que a referida nos estudos mencionados.

Considerando-se a relativamente vasta gama de patologias que foram evidenciadas pelos estudos experimentais e epidemiologicos, os achados são preocupantes e devem merecer a atenção das autoridades de saúde pública.

Um fato que deve merecer especial preocupação é de que os PCBs foram, no passado, utilizados extensivamente pela indústria elétrica e eletrônica brasileiras; além disso, ma- 
terial elétrico importado deve conter grandes quantidades de PCBs. Todo esse material é de vida relativamente longa e, à medida que forem sendo desativados, os PCBs nele contidos devem ser dispostos de maneira adequada.

Como já referido, em janeiro de 1981, Portaria Interministerial proibiu, no Brasil, a fabricação e a comercialização dos PCBs, assim como a sua disposição em cursos e coleções de água ou em locais expostos a intempéries, mas não existem normas estabelecidas para que essa disposição seja feita de tal maneira que impeça a contaminação ambiental. E de se supor, portanto, que equipamento elétrico contendo PCBs esteja sendo desmontado ou disposto sem as mínimas preocupações quer quanto às pessoas que fazem esse serviço, quer em relação à proteção do meio ambiente.

Se o governo canadense ${ }^{16}$ estimava em 1982 que os equipamentos que contém PCBs somente deixarão de ser usados nos próximos 40 anos, é de se imaginar que no Brasil, onde é mínima a preocupação por este risco, equipamentos contendo PCBs ainda serão usados por muitas décadas.

Impõe-se, portanto, a adoção imediata pelo governo brasileiro de normas rígidas quanto à disposição dos PCBs e dos equipamentos, quer elétricos quer de outra natureza, que os contenham. Sabe-se que desde a proibição da fabricação e uso dos PCBs no Brasil numerosas indústrias dispóem de estoques de PCBs que devem ser dispostos de alguma maneira. Sabe-se que, pelo menos, uma grande indústria brasileira está instalando equipamento para incineração de produtos com resíduos clorados, entre os quais incluem-se os PCBs. Estas instalações que utilizam técnica moderna e adequada para disposição destes produtos poderão ser usadas posteriormente, por outras grandes empresas que mantêm estoques de PCBs à espera de uma solução para sua disposição.

A dificuldade maior reside nas pequenas empresas que mantém estoques de PCBs e cujos responsáveis não têm conhecimento dos reais riscos do produto. Tais empresas, possivelmente, descartar-se-ão dos PCBs da maneira mais fácil. A simples proibição do lançamento dos PCBs em cursos e/ou coleções de água prevista pela legislação vigente não terá nenhum valor caso não haja fiscalização que, como se sabe, é precária e de difícil execução.

Finalmente, são indispensáveis estudos sistemáticos sobre resíduos de $\mathrm{PCBs}$ em alimentos, provavelmente a mais importante via de con- taminação do organismo dos brasileiros. Para isso, os laboratórios de Saúde Pública deveriam estar equipados para efetuar a análise dos residuos de PCBs em alimentos, como é feito em relação aos resíduos de inseticidas organoclorados; além disso, impõe-se um estudo das embalagens dos alimentos, possível fonte de contaminação dos mesmos. Não deve ser esquecida a urgente necessidade de se estudar os resíduos de PCBs em leite humano, estudo tanto mais importante por ter se verificado os elevados níveis de acúmulo desses produtos no tecido celular subcutâneo das mulheres estudadas.

\section{CONCLUSOEES}

Do que foi apresentado, as seguintes conclusões são de importância:

1 - Na amostra dos habitantes do Grande São Paulo, 12,7\% apresentam acúmulo de PCBs acima de $1,95 \mathrm{ppm}$, valor apreciavelmente maior que o observado em outros países altamente industrializados.

2 - As mulheres apresentam, em média, valores de acúmulo superiores aos dos homens.

3 - No sexo masculino nota-se a tendência de aumento do acúmulo à medida que aumenta a idade.

\section{RECOMENDAÇOES}

1 - Não obstante a proibição da fabricação e do uso dos PCBs em território brasileiro, grande número de equipamentos elétricos ainda existe em uso e este terá ainda a duração de algumas dezenas de anos, constituindo risco permanente de contaminação ambiental.

2 - A simples proibição legal de não dispor os PCBs em cursos e/ou coleções d'água não é suficiente para a preservação ambiental: impõe-se urgentemente o estabelecimento de normas técnicas para a destruiçăo eficiente desses produtos e a fiscalização da sua execução.

3 - Há necessidade de ser feito estudo epidemiológico sistemático que estude os efeitos do acúmulo de PCBs sobre a saúde dos brasileiros.

4 - Tornam-se de grande importância para a Saúde Pública brasileira que a análise sistemática de resíduos de PCBs em alimentos e suas respectivas embalagens seja levada a cabo pelos órgãos competentes de Saúde Pública com o fito de se verificar a importância 
dessa via de entrada para estes compostos no organismo da população brasileira; tal análise deverá ser seguida de medidas de controle que impeçam a contaminação da cadeia alimentar pelos PCBs.

5 - Tendo em vista os níveis elevados de acúmulo dos PCBs no organismo das mulheres, é de grande importância que seja feito um estudo dos teores em PCBs do leite humano.

\section{AGRADECIMENTOS}

Os autores desejam apresentar seus agradecimentos ao Instituto Médico Legal, da Secre- taria de Segurança Pública do Estado de São Paulo, na pessoa do seu ex-Diretor Dr. Harry Shibata e do seu atual Diretor, Dr. Rubens Maluf pela permissão para a colheita de amostras de tecido celular subcutâneo; a Maria Aparecida Rezende de Araújo, Adelaide Rosas Forte e Maria Helena Callera Pedrosa pelo auxílio na execução das análises cromatográficas; ao Programa de Disseminação Seletiva de Informação da Biblioteca da Faculdade de Saúde Pública da Universidade de São Paulo pelo acesso à literatura científica sobre policloretos de bifenila e à Bibliotecária Angela Maria Belloni Cuenca pela revisão das citações bibliográficas.

NOGUEIRA, D. P. et al. [Accumulation of polychlorinated biphenyls in the population of Greater S. Paulo, Brazil]. Rev. Saúde públ., S. Paulo, 21:279-90, 1987.

\begin{abstract}
Experimental and clinical studies have proved, conclusively, that polychlorinated biphenyls (PCBs) can effect human health adversely, to the point of constituting a serious public health problem. Thus the study of the deposition of PCBs in a significant sample of the inhabitants of Greater S. Paulo, Brazil, was considered to be of interest. Samples of subcutaneous fat were obtained from 189 people who had suffered violent death and analysed by as chromatography. Average levels of 0.8661 parts per million (ppm) were found in males, who presented a linear increase of deposition according to chronological age; this was not observed with females, however in whom the values of deposition were, in general, $1.4530 \mathrm{ppm} 12.7 \%$ of all samples presented values greater than $1.95 \mathrm{ppm}$. The importance of such findings is discussed and protective measures that should be taken to avoid further increase of absortion by the Brazilian population are indicated.
\end{abstract}

UNITERMS: Polychlorobiphenyl compounds, analysis. Environmental pollution. Food contamination, chemical.

\title{
REFERENCIAS BIBLIOGRÁFICAS
}

1. ALENCASTRO, L. F. de; PRELAZ, V.; TARRADELLAS, J. Contamination of silos in Switzerland by PCB residues in coatings. Bull. environ. Contam. Toxicol., 33:270-6, 1984 .

2. ANDERSON, H. A. Utilization of adipose tissue biopsy in characterizing human halogenated hydrocarbon exposure. Environ. Hlth Perspect., 60:121-31, 1985.

3. AURELICH, R. J.; BURSIAN, S. J.; BRESLIN, N. W. J.; OSLON, B. A.; RINGER, R. K. Toxicolegical manifestation of $2,4,5$, $2^{\prime}, 4^{\prime}, 5^{\prime}-2,3,6,2,3^{\prime}, 6$, - and 3,4 , $5,3,4,4$, , - hexachlorobiphenyl and Aroclor 1254 in minks. J. Toxicol. environ. Hlth, 15:63-79, 1985.

4. BAJULA, G.; HERNANDEZ, L. M.; GONZÁLEZ, M. J.; RICO, M. C. Presence of organochloride pesticides, polychlorinated biphenyls, and mercury in Spanish human milk samples. Bull. Environ. Contam. Toxicol., 28:573-7, 1982.

5. BENNETT, G. A.; DINKER, C. K.; WARREN, M. F. Morphological changes in the livers of rats resulting from exposure to cer- tain chlorinated hydrocarbons. J. industr. Hyg. Toxicol., 20:98-123, 1938.

6. BERG, O. W.; DIOSADY, P. L.; REES, A. Columm chromatographic separation of polychlorinated biphenyls from chlorinated hydrocarbons pesticides and their subsequent gas chromatographic quantitatives in terms of derivatives. Bull. environ. Contam. Toxicol., 7:338-42, 1972.

7. BERGLUND, F. Levels of polychlorinated biphenyls in foods in Sweden. Environ. Hith Perspect., 1:67-71, 1972.

8. BERQUÓ, E.; SOUZA, J. M. P. de; GOTLIEB, S. L. D. Bioestatísica. São Paulo, E.P.U., 1981.

9. BICKEL, M. H. \& MUEHLBACH, S. Pharmacokinetics and ecodisposition of polyhalogenated hydrocarbons: aspects and concepts. Drug Metab. Rev., 11:149-90, 1980.

10. BROWN, R. W.; ROSS, M. R.; FEINGOLD, J.; BROWN Jr., J. Effects of PCB exposure on biochemical and hematological findings in capacitor workers. Environ. Hlth Perspect., 60:165-84, 1985. 
11. BUSH, B.; SNOW, J.; CONNOR, S.; KOBLINTZ, R. Polychlorinated biphenyls congeners (PCBs) p, p' - DDE and hexachlorobenzene in human milk in three ares of upstate New York. Arch. environ. Contam. Toxicol., 14:443-50, 1985.

12. CARTER, J. W. Effects of dietary PCBs (Aroclor 1254) on serum levels of lipoprotein chloresterol in Fisher rats. Bull. environ. Contam. Toxicol., 34:427-31, 1985.

13. CHASE, K. H.; WONG, O.; THOMAS, D.; BERNEY, B. W.; SIMON, R. K. Clinical and metabolic abnormalities associated with occupational exposure to polychlorinated biphenyls. J. occup. Med., 24:109-14, 1982.

14. CHIA, L.-G. \& CHU, F.-L. Neurological studies on polychlorinated biphenyl (PCB) poisoned patients. Amer. J. industr. Med., 5:117-26, 1984.

15. DE KOK, A. GEERDINK, R. B.; FREI, R. W.; BRINKMAN, A. A. T. Limitation on the use of perchlorination as a technique for the quantitative analysis of polychlorinated biphenyls. Int. J. environ. anal. Chem., 11:17-41, 1981 .

16. DEPARTMENT OF ENVIRONMENT. Canada. Guidelines for the management of PCBs wastes. Can. Gazette, (Pt. 1): 9237-8, Dec. $11,1982$.

17. ERNEY, R. D. Rapid screening method for analysis of chlorinated pesticides and chlorinated biphenyl residues in fish. J. Amer. Off. Ass. Chem., 57:576-9, 1974.

18. FALANDYSZ, J. \& SZEFER, P. Chlorinated hydrocarbons in diving ducks wintering in Gdanski Bay, Baltic Sea. Sci. Total Environ., 24:119-27, 1982.

19. FIGUEIREDO. A.; LAVORINO, P. R.; PARRA, J. M. P. Askaréis: risco, controles, manuseio e destinação final. Rev. bras. Saúde ocup., 8(32):20-7, 1980.

20. FINKLEA, J.; PRIESTER, L. E.; CREASON, T. H.; HAUSER, T.; IHNNERS, T.; HAMMER, D. I. Polychlorinated biphenyl residues in human plasm expose a major urban pollution problem. Amer. J. publ. Hlth, 62: $645-51,1972$

21. GUZELIAN, P. S. Clinical evaluation of liver structure and function in humans exposed to halogenated hydrocarbons. Environ. Hlth Perspect., 60:159-64, 1985.

22. HANSEN, L. G.; WASHKO, P. W.; TUINSTRA, L. G. M.; DORN, S. B.; HINESLY, T. D. Polychlorinated biphenyl pesticide and heavy metal residues in swine foraging on sewage sludge amended soils. J. agric. Food Chem., 29:1012-7, 1981.

23. IMANISHI, J.; OKU, T.; OISHI, K.; KISHIDA, T.; NOMURA, H.; MIZUTANI, T. Reduced resistance to experimental viral and bacterial infections of mice treated with polychlorinated biphenyl. Biken J., 27:195-8, 1984.
24. JACOBSON, S. W.; FEIN, G. G.; JACOBSON, J. L.; SCHWARTZ, P. M.; DOWLER, J. K. The effect of intrauterine PCB exposure on visual recognition memory. Child Dev., 56:853-60, 1985.

25. JENKE, H. S. Polychlorinated biphenyls interfere with the regulation of hydroxymethylglutaryl-coezime $\mathbf{A}$ reductase activity in rat liver via enzyme-lipid intereraction and the transcriptional level. Biochem. biophys. Acta, 837:85-93, 1985.

26. JENSEN, S.; JOHNELS, A. G.; OLSSON, M.; OTTERLIND, G. DDT and PCB in marine animals from Swedish waters. Nature, 224: 247-50, 1969.

27. JONSSON, H. T.; WALKER Jr., E. M.; GREENE, W. B.; HUGHSON, M. D.; HENNIGAR, G. R. Effects of prolonged exposure to dietary DDT and PCB on rat liver morphology. Arch, environ. Contam. Toxicol., 10:171-83, 1981 .

28. KIMBROUGH, R. D.; LINDER, R. E.; GAINES, T. B. Morphological changes in livers of rats feed polychlorinated biphenyls. Arch. environ. Hlth, 25:354-64, 1972.

29. KREISS, K. Studies on populations exposed to polychlorinated biphenyls. Environ. Hlth Perspect., 60:193-9, 1985.

30. KREISS, K.; ROBERTS, C.; HUMPHEREY, H. E. B. Serial PBB levels, PCB levels and clinical chemistries in Michigan's PBB cohort. Arch. environ. Hlth, 37:141-7, 1982.

31. KURATSUNE, M.; YOSHIMURA, T.; MATSUZAKA, J.; YAMAGUCHI, A. Yusho a poisoning caused by rice oil contaminated with polychlorinated biphenyls. HSMHA Hlth Rep., 36:1083-91, 1971.

32. LAWTON, R. W.; ROSS, M. R.; FEINGOLD, J. Spirometric findings in capacitor workers occupationally exposed to polychlorinated biphenyls (PCBs). [American Occupational Health Conference, Kansas City, MO, April 17,1985 - in press].

33. LLOYD, J. W.; MOORE, R. M.; WOOLF, B. S.; HARVEY, P. S. Polychlorinated biphenyls. J. occup. Med., 18:109-13, 1976.

34. LUOTAMO, M.; JARVISALO, J.; AITIO, A. Analysis of polychlorinated biphenyls (PCBs) in human serum. Environ. Hlth Perspect., 60:327-32, 1985.

35. Mac LEOD, K. E. Polychlorinated biphenyl in indoor air. Environ. Sci. Technol., 5:926-8, 1981.

36. MARKS, T. A.; KIMMEL, G. L.; STAPLES, R. E. Influence of symetrical polychlorinated biphenyl isomers on embryo and fetal development in mice. Toxicol. appl. Pharmacol., 61:269-76, 1981 .

37. MES, J.; DAVIES, D. J.; TURTON, D. Polychlorinated biphenyl and other hydrocarbon residues in adipose tissue of Canadians. Bull. environ. Contam. Toxicol., 28:97-104, 1982. 
38. MILLER, J. W. Pathologic changes in animals exposed to a commercial chlorinated diphenyl. Publ. Hlth Rep., 59:1085-93, 1944.

39. MILLER, R. W. Congenital PCB poisoning: a revaluation. Environ. Hlth Perspect., 60:211-4, 1985.

40. MONTGOMERY, D. C. \& PECK, E. A. Introduction to linear regression analysis. New York, John Wiley \& Sons, 1982.

41. MORGAN, R. W.; WARD, J. M.; HARTMAN, P. E. Aroclor 1254 induced intestinal metaphasic and adenacarcinoma in the glandular stomachs of F344 rats. Cancer Res., 41: 5052-9, 1981.

42. MORIARTY, F. Organochlorine inseticides. London, Academic Press, 1975. p. 21-27.

43. NEAL, R. A. Mechanisms of the biological effects of PCBs, polychlorinated dibenzo-p-dioxins and polychlorinated dibenzofurans in experimental animals. Environ. Hith Perspect., 60:41-6, 1985 .

44. NISBET, I. C. T. \& SARUFIM, A. F. Rates and routes of transport of PCBs in the environment. Environ. Hlth Perspect., 1:21-38, 1972.

45. O'KEEFE, P. W.; SILKWORTH, J. B.; GIERTHY, J. F.; SMITH, R. M.; De CAPPRIO, A. P.; TURNER, J. N.; EADON, G.; HILKER, D. R.; ALDOUS, K. M.; KAMINSKY, L. S., COLLINS, D. N. Chemical and biological investigation of a transformer at Binghamton, NY. Environ. Hlth Perspect., 60: 201-9, 1985.

46. OUW. H. K.; SIMPSOM, G. R.; SIYALI, D. S. Use and health effects of Aroclor 1242, a polychlorinated biphenyl, in an electrical industry. Arch. environ. Hlth, 31:189-94, 1976.

47. P.ACHECO, L. A. A. M. PCB - policloretos de bifenila. São Paulo, Departamento de Saúde Ambiental da Faculdade de Saúde Pública da USP, s.d. 6p. [Apostila mimeografada].

48. POLISHUK, Z. W.; RON, M.; WASSERMANN, M.; CUCOS, S.; WASSERMANN, D.; LEMESCH, C. Pesticides in people. Pesticides Monit. J., 10:121-9, 1977.

49. PRESTON, B. D.; MILLER, J. P.; MOORE, R. W.; ALLEN, J. R. Promoting effects of polychlorinated biphenyls (Aroclor 1254) and polychlorinated dibenzofuran-free Aroclor 1254 on diethylnitrosamine-induced tumorogenesis in the rat. J. nat. Cancer Inst., 66:509-15, 1981.

50. PRICE, H. A. \& WELCH, R. L. Occurence of polychlorinated biphenyls in humans. Environ. Hlth Perspect., 1:73-7, 1972.

51. PROCTOR, N. H. \& JAMES, P. Chemical hazardous of the workplace. Philadelphia, Lippincortt, 1978. p. 165-7.

52. RIFKIND, A. B.; SASSA, S.; REYES, J.; MUSCHICK, H. Polychlorinated aromatic hydrocarbon lethality, mixed-function oxidase induction, and uroporphyrinogen decarboxylase inhibition in the chick embryo: dissociation of dose-reponse relationships. Toxicol. appl. Pharmacol., 78:268-79, 1985.

53. ROBOZ, J.; GREAVES, J.; BEKESI, J. G. Polybrominated biphenyls in model and environmentally contaminated human blood. $E n$ viron. Hlth Perspect., 60:107-13, 1985.

54. SCHECTER, A. \& TIERNAM, T. Occupational exposure to polychlorinated dioxins, polychlorinated furans, polychlorinated biphenyls and bipheniles after an electrical panel and transformer accident in an office building in Binghamton, NY. Environ. Hlth Perspect., 60:305-13, 1985 .

55. SHECTER, A.; TIERMAM, T.; SCHAFFNER, F.; TAYLOR, M.; GITLITZ, G.; Van NESS, G. F.; GARRET, J. H.; WAGEL, D. J. Patient fat biopsis for chemical analysis and liver biopsis for ultrastructural characteriza. tion after exposure to polychlorinated dioxins, furans and PCBs. Environ. Hlth Perspect., 60:241-54, 1985.

56. SCHWARTZ, L. Dermatites from synthetic resins and waxes. Amer. J. publ. Hlth, 26: 586-92, 1936.

57. SEPPÄLÄINEN, A. M.; VUOJOLAHTI, P.; ELO, O. Reversible nerve lesions after accidental polychlorinated biphenyl exposure. Scand. J. Wk environ. Hlth, 11:91-5, 1985.

58. SLEIGHT, S. Effects of PCBs and related compounds on hepatocarcinogenesis in rats and mices. Environ. Hlth Perspect., 60:35-9, 1985.

59. SMITH, A. J. Polychlorinated biphenyls. In: Bennet, G. F.; Feats, F. S. \& Wilder, I. Hazardous materials spills handbooks. New York, McGraw-Hill, 1982.

60. TAYLOR, P. R.; LAWRENCE, C. E.; HWRANG, H. L.; PAULSON, A. S. Polychlorinated biphenyls: influence on birthweight and gestation. Amer. J. publ. Hlth, 74:1153-4, 1984.

61. TOMBERGS, H. P. The PCB situation in Germany. Environ. Hlth Perspect., 1:179-80, 1972.

62. WARD, J. M. Proliferative lesions of the glandular stomach and liver in F344 rats fed diets containing Aroclor 1254. Environ. Hlth Perspect., 60:89-95, 1985.

63. WASSERMANN, M.; NOGUEIRA, D. P.; TOMATIS, L.; MIRRA, A. P.; SHIBATA, H.; ARIE, G.; CUCOS, S.; WASSERMANN, D. Organochlorine compounds in neoplastic and adjacent apparently normal breast tissue. Bull. environ. Contam. Toxicol., 15:478-84, 1976.

64. WASSERMANN, M.; BERCOVICI, B.; CUCOS, S.; WASSERMANN, D.; RON, M.; Storage of some organochlorine compounds in toxemia of pregnancy. Environ. Res., 22:404-11, 1980.

65. WASSERMANN, M.; RON, M.; BERCOVICI, B.; WASSERMANN, D.; CUCOS, S.; PINES, 
A. Premature delivery and organochlorined compounds: polychlorinated biphenyls and some organochlorine inseticides. Environ. Res., 28:106-12, 1982.

66. WHITE, R. J.; KIM, H. M.; KIM, J. S. PCBs in striped bass collected from the Hudson River, New York, during fall, 1981. Bull. environ. Contam. Toxicol., 34:883-9, 1985.

67. WICKZER, T. M. \& BRILLIANT, L. B. Testing for polychlorinated biphenyls in human milk. Pediatrics, 68:411-5, 1981.

68. WOLFF, M. S.; THORNTON, J.; FISCHBEIN, A.; LILIS, R.; SELIKOFF, I. J. Disposition of polychlorinated biphenyls congeners in occupational exposed persons. Toxicol. appl. Pharmacol., 62:294-306, 1982.

69. WOOD, N. F. Extraction and clean-up of organochlorine pesticide residues by colum chromatography. Analyst, 94:339-405, 1969.
70. WORLD HEALTH ORGANIZATION. Polychlorinated biphenyl and terphenyls. Geneva, 1976. (Environmental Health Criteria, 2).

71. YAGI, N. \& ITOKAWA, Y. Lipid metabolism in polychlorinated biphenyl-poisoned rats. $E_{n-}$ viron. Res., 22:139-44, 1980.

72. YAKUSHI, T.; WATANABE, I.; KUWABARA, K.; TANAKA, R.; KASHIMOTO, T.; KUNITA, N.; HARA, I. Postnatal transfer of PCBs from exposed mothers to their babies: influence of breastfeeding. Arch. environ. Hith, 39:368-75, 1984.

73. YOBS, A. R. Levels of polychlorinated biphenyls in adipose tissue of the general population of the nation. Environ. Hlth Perspect., 1:79-81, 1972.

Recebido para publicação em 16/2/1987 Reapresentado em 27/5/1987

Aprovado para publicação em 4/6/1987 